Laser Chem. 1986, Vol. 6, pp. 1-14

0278-6273/86/0601-0001\$12.00/0

(C) harwood academic publishers gmbh

Printed in Great Britain

\title{
ELECTRON TRANSFER COLLISIONS OF EXCITED SODIUM ATOMS AND OXYGEN MOLECULES
}

\author{
C. DESFRANCOIS, J.P. ASTRUC, R. BARBE, \\ A. LAGREZE, J.P. SCHERMANN
Laboratoire de Physique des Lasers, Associé au C. N. R. S. Université Paris-Nord 93430 VILLETANEUSE, FRANCE \\ Centre scientifique et Polytechnique,
}

\section{ABSTRACT}

The vibronic network model is here considered for excited sodium atoms and oxygen molecules collisions. Electronic to vibration transfer and reaction cross sections are computed with a single adjustable parameter: the ionic covalent matrix element. The comparison between the atom-molecule and the quasi-free electron models is presented.

\section{INTRODUCTION}

The electron transfer or harpoon mechanism is well known for the reaction of alkali atoms (1), chemiluminescence or chemiionization processes (2). When the alkali atoms are excited and collide with electron accepting molecules, the electronic to vibration energy transfer process (or quenching) competes with the reactive process, both go- 
verned by the electron transfer which exibits the following trend in the case of halogen molecules (3) : the electron transfer cross sections increase from ground states of the alkali atoms to the first resonant states due to the larger internuclear crossing distances between covalent and ionic states, then fall rapidly to zero for second resonant states and above.

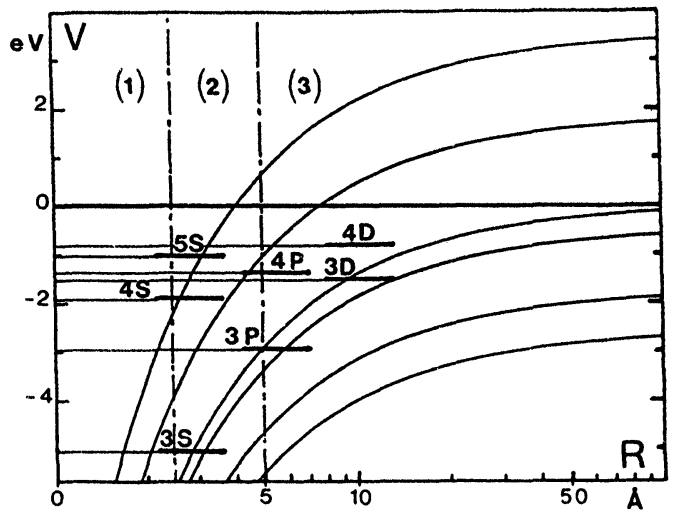

Pigure 1: Ionic-covalent curve crossings between excited states of sodium and molecules. Region ( 3 ) corresponds to negative molecular ions unperturbed by the presence of the $\mathrm{Na}^{+}$ core. For $\mathrm{Na}+\mathrm{O}_{2}$, the ionic intermediate model is stricly valid for the $3 P$ level and approximate for the 35 level.

The electron transfer process depends upon the overlap of the orbitals of the exchanged electron in the donor atom and the accepting molecule. If the donor atom is highly excited, its valence electron cloud has a lar- 
ge size and the overlap with that of a strongly bound molecular negative ion will be rather poor. This is the case for halogens with electron affinities in between 1 and $3 \mathrm{eV}$. By contrast, molecules which posess very small vertical affinities and, thus, attach low energy electrons. will be of interest in collisions with excited atoms since the overlap will be more favorable due to the large spatial extension of the outer electron orbital of their negative ions.

Collisions of excited sodium atoms with oxygen molecules are here considered. The oxygen molecule has a rather small adiabatic electron affinity of $.44 \mathrm{eV}$ and a very small vertical affinity corresponding to molecular negative ions $\mathrm{O}_{2}^{-}$loosely bound as compared to halogen negative ions. Experiments have been performed to measure the electronic to vibration energy transfer of the first resonant $3 P$ state of sodium by oxygen molecules (4)(5) as well as reactive cross section of the $3 \mathrm{P}, 5 \mathrm{~S}$ and $4 \mathrm{D}$ states (6).

A complete interpretation would require a calculation of the potential energy surfaces and their couplings, followed by trajectory computations on these surfaces. In the case of the reacting $D$ state of sodium, for example, this would require dynamical studies over several tens of surfaces of different symmetries. The simpler approach of Bauer, Fischer and Gilmore (BFG) (7) was chosen. The validity of this model in the case of $\mathrm{Na}^{-\mathrm{O}_{2}}$ collisions is examined in chapter II and the used atomic and molecular data are given. The results of this model are compared in chapter III to the experimental data of Barker $(10)$ and 
Hertel (4) for the quenching cross sections of the 3P state. The model is extended to the inter multiplet mixing cross sections and to the reactive cross sections in chapter llV. In this last case, a comparison is given between this multiple crossing model and the quasi-free electron model.

II. IONIC COVALENT CURVE CROSSING MODEL

INCLUDING THE VIBRATIONAL DEGREE OF FREEDOM

\section{A/ Principle}

Two sets of states $\left|\phi_{c} v\right\rangle$ and $\left|\Phi_{i} v^{\prime}\right\rangle$ corresponding respectively to the covalent $\mathrm{Na}$ $+\mathrm{O}_{2}(\mathrm{v})$ and ionic $\mathrm{Na}^{+}+\mathrm{O}_{2}^{-}\left(\mathrm{v}^{\prime}\right)$ systems, are considered. These sets are only coupled by potential coupling terms

$$
\left\langle\Phi_{i} v^{\prime}|H| \phi_{c} v^{\prime}\right\rangle=H_{12} .\left\langle v \mid v^{\prime}\right\rangle
$$

At each curve crossing, a diabatic transition probability $P_{12}$ is given by the Landau zener expression

$$
P_{12}=\exp \left[-2 H_{12}^{2}\left\langle v \mid v^{\prime}\right\rangle / \hbar v_{r} \Delta S_{12}\right]
$$

where $v_{r}$ is the radial velocity at the crossing point and

$$
\Delta S_{12}=\left|\frac{d V_{i}\left(R_{c}\right)}{d R}-\frac{d V_{c}(R)}{d R}\right|
$$

The entrance channel is one of the covalent curve corresponding to an excited atom in one of the $(n, 1)$ state, with an approach angle $\theta$ in the molecular frame and an oxygen molecule in the ${ }^{3} \Sigma_{g}^{-}$and $v=0$ ground $s$ tate. Due to the centrifugal barrier, the relative motion will be reversed at the turning point and the representating point will end up in one of the exit channels, generally an excited sodium atom in another $\left(n^{\prime}, l^{\prime}\right)$ state with an oxygen molecule in one of the three electronic states 
${ }^{3} \Sigma_{g},{ }^{1} \Delta_{g}$ and ${ }^{1} \Sigma_{g}^{+}$. At thermal energies here considered, ion pair formation is energetically forbidden.

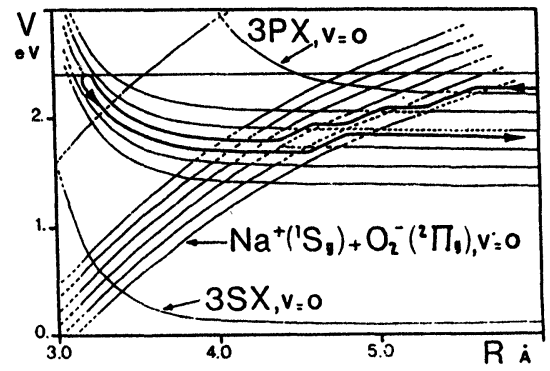

Figure 2a: In the vibronic network, motion of the $\mathrm{Na}_{-} \mathrm{O}_{2}$ representating point for electronic to vibration transfer.

The representating point on the vibronic network may end up on an ionic curve after the first passage. The exit channel is then energetically forbidden and the representating point turns back towards another classical turning point. This is equivalent to a classical trajectory leading to complex formation (10).

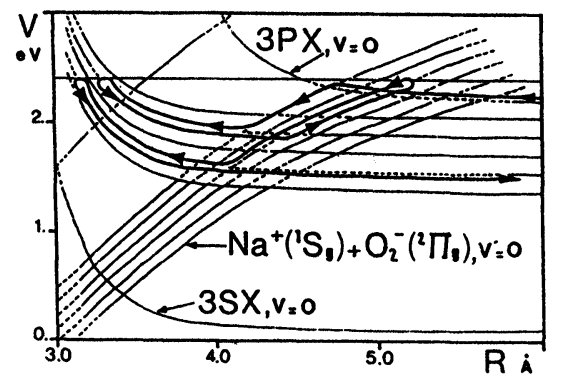

Fiqure 2b : Complex formation 
The representating point may also appear on an ionic curve below the lowest $(3 S, v=0)$ covalent curve.

Reaction will then occur or not whether it is energetically allowed or not, and whether or not these initial dynamical conditions are favorable.

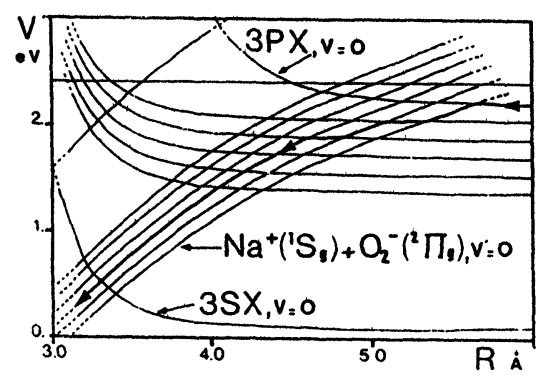

\section{Figure 2c: Reaction}

The validity of this many channel model of non adiabatic coupling has been examined by Child and Baer, and Klomp and Los. The BFG model does not allow for interferences between different paths in the vibronic network and requires a loss of memory of the vibrational phases between each crossing. For the excited sodium atom-oxygen molecule collisions at thermal energies here considered, the classical times between two electronic crossing are longer than $2.10^{-14} \mathrm{~s}$, vibrational period of $\mathrm{O}_{2}$, justifying the use of the BFG model.

For molecules such as $\mathrm{N}_{2}$ or $\mathrm{H}_{2}$ with negative electron affinities, the ionic intermediate model, corresponding to crossings bet- 
ween ionic and covalent curves, has been compared to ab initio surface calculations. The internuclear crossing distances are of the order of magnitude of the atom and molecule sizes. The charge transfer between the excited atom and the molecule still exists, but the potential energy curves are very distorted as compared to the negative molecular ion curves.

on the contrary, for molecules with positive electron affinities, the crossings between ionic and covalent systems take place at large internuclear distances. From ab initio calculations on alkali-halogen systems, one can infer that the negative molecular ion curves are not perturbed by the presence of the $\mathrm{Na}^{+}$core at internuclear distances larger than $10 \AA$. The ionic model is thus probably valid for sodium energy levels above the $3 P$ state.

\section{B/ Vibronic network}

The covalent potential curves are approximated by Lennard Jones potential curves.

$$
v_{c}(R)=-\frac{C_{6}}{R^{6}}+\frac{C_{12}}{R^{12}}-\left[I P(n, 1)-E A\left(v, v^{\prime}\right)\right]
$$

where IP $(n, 1)$ is the ionization potential of the considered excited state $(n, 1)$ of the sodium atom, EA(v, $\left.v^{\prime}\right)$ the vertical affinity of oxygen between the $v$ level of $\mathrm{O}_{2}$ and $v^{\prime}$ level of $\mathrm{O}_{2}^{-}$. The $\mathrm{C}_{6}$ coefficients are. given by

$$
C_{6}=2 \alpha_{O_{2}} n^{* 2}\left[5 n^{2}+1-31(1+1)\right]
$$

where the oxygen polarizability is equal to $1.59 \AA^{3}$ and $n$ is the effective quantum number of state $(n, 1)$. 
The $\mathrm{C}_{12}$ coefficients are given by

$$
C_{12}=(.9 \pm .3) 10^{-16} \cdot\left(P_{\mathrm{Na}}+P_{\mathrm{O}_{2}}\right) \text { erg }
$$

where $\mathrm{P}_{\mathrm{Na}}$ and $\mathrm{P}_{2}$ are the electronic radii of $\mathrm{Na} *$ and $\mathrm{O}_{2}$.

The expression of the potential energy of the ionic system is given by

$$
V_{i}(R)=-\frac{e^{2}}{4 \pi \epsilon \cdot R}-\frac{\left(\alpha_{O_{2}^{-}}+\alpha_{N a}\right)}{4 \pi \epsilon \cdot 2 R^{4}}
$$

where $\alpha_{0_{2}^{-}}=2.64 \AA^{3}$ and $\alpha_{\mathrm{Na}^{+}}=.18 \AA^{3}$

The Franck Condon factors $\left\langle v \mid v^{\prime}\right\rangle$ between the $\mathrm{O}_{2}+$ e and the $\mathrm{O}_{2}^{-}$systems are computed with $\mathrm{O}_{2}$ and $\mathrm{O}_{2}^{-}$potential curve Morse expressions. The autodetachment process of the $\mathrm{O}_{2}^{-}$ion above $\mathrm{v}^{\prime}=3$ is ignored since it corresponds to a time scale $10^{-10}-10^{-12} \mathrm{~s}$ much longer than the mean collision time $\left(10^{-13} \mathrm{~s}\right)$.

The only adjustable parameter which is used here is the ionic covalent coupling matrix element $\mathrm{H}_{12}$. Following Smirnov, olson and Los, this $\mathrm{H}_{12}$ matrix element can be written, in reduced form, as

( 8$) \quad H_{12}^{*}=A \cdot R_{c}^{*} \cdot \exp \left(-B \cdot R_{c}^{*}\right)$

where

$$
\begin{aligned}
& H_{12}^{*}=H_{12}\left(R_{c}\right)\left(\operatorname{IP}(n, 1)-E A\left(v, v^{\prime}\right)\right)^{1 / 2} \\
& R_{c}^{*}=R_{C}(2 . \operatorname{IP}(n, 1))^{1 / 2}
\end{aligned}
$$

The ionic-covalent crossing distance $R_{c}$ is given by $V_{i}\left(R_{c}\right)=V_{c}(R)$.

From a simple LCAO calculation extending to highly excited states the method of Grice and Herschbach (9), we deduced the following numerical values of the $A$ and $B$ coefficients $: A=1.73, B=.7$ corresponding to 
the electron affinity of $\mathrm{O}_{2}$. Several values of B were tried in order to fit the available experimental datas keeping the A coefficient constant.

Following Gislason (1), a simple estimate of the angular dependance of the ionic covalent coupling element upon the angle between the collision axis can be deduced from the respective symmetry properties of the covalent and ionic atom-molecule surfaces :

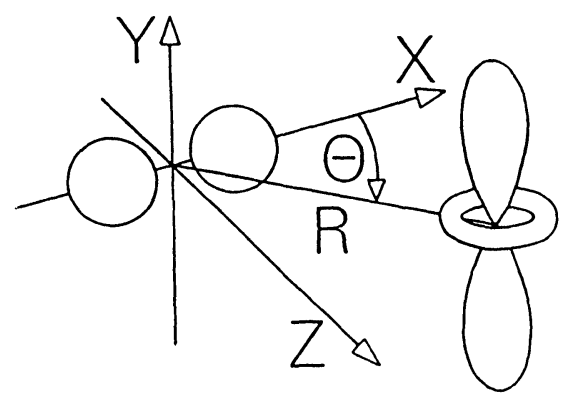

Piqure 3 : Sodium-oxygen approach configuration

one combines the symmetry states of $\mathrm{Na}$ with those of $\mathrm{O}_{2}$ in the three configurations corresponding to the three symmetry group $\mathrm{C}_{\infty v}$, $\mathrm{C}_{s}$ and $\mathrm{C}_{2 \mathrm{v}}$. When compared to the combined symmetries of $\mathrm{Na}$ and $\mathrm{O}_{2}$, two states are coupled or not whether they belong or not to the same irreducible representation. The $\mathrm{H}_{12}$ matrix elements are then approximated by

(11) $\quad H_{12}(R, \theta)=H_{12}(R) \quad F(\theta)$

where $F(\theta)$ is a Legendre polynomial. 
II. QUENCHING OF THE 3P STATE OF SODIUM

BY OXYGEN MOLECULES.

The electronic to vibration energy transfer in collisions between the resonant $3 p$ state of sodium by several molecules has been studied in beam experiments by Hertel (4) and Blais (5). By measuring the kinetic energy loss of the sodium atom after collision with a molecule, Hertel et al have determined the variation of the quenching cross section $Q_{q}$ of the $3 \mathrm{P}$ state of $\mathrm{Na}$ as a function of the kinetic energy $E_{c m}$ (in the center of mass frame) after collision. The experimental curve shown in figure (4) represents the energy dependance of the differential cross section $\left(\frac{d Q_{q}}{d E}\right) . E_{c m}$.

The entrance channel $\mathrm{Na}(3 \mathrm{P})+\mathrm{O}_{2}\left({ }^{3} \Sigma_{g}^{-}\right)$ corresponds to the three electronic states (with spin multiplicities 2 or 4 ) according to the three orbitals $p_{x}, p_{y}, p_{z}$ of the sodium atom. Two cases are here considered in the molecular (and not the laboratory) frame: an equal mixture of the three $p$ orbital of sodium or separately, the three $p_{x}, p_{y}$ and $p_{z}$ orbitals.

An alternative to the Landau Zener expression of the probability of the BFG model has been proposed by Barker, taking into account trajectories which are classicaly allowed on the lower adiabatic potential energy curves, but classicaly forbidden on the diabatic curves. The two expressions of the transition probabilities (BFG or Barker) have been tested and give very nearly the same results.

The best fit of the experimental data i.e. the total quenching cross section (10) 
and the energy differential cross section (4) are displayed on figure 4 .

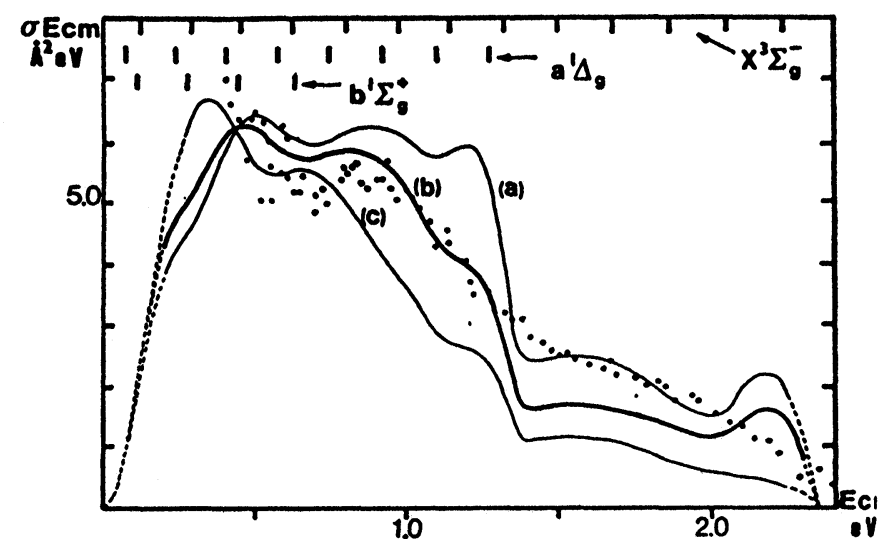

Fiqure 4: Differential cross section for the quenching of the $3 P$ state of sodium by oxygen molecules. Dots are experimental (reference 6). Curve (a) corresponds to the ionic-covalent $\mathrm{H}_{12}$ matrix element of olson (ref 20), (b) to a $B$ value of . 8, (c) to a B value of. 7 .

The total quenching cross section does not appreciably vary for incident energies below $.27 \mathrm{eV}$ which corresponds to the energy threshold of the reaction

(12) $\mathrm{Na}^{*}(3 \mathrm{P})+\mathrm{O}_{2} \rightarrow \mathrm{NaO}+\mathrm{O}$

The influence of the values of the $C_{6}$ and $C_{12}$ coefficients of the covalent potential (equations 5 and 6 ) has been tested by dividing these values by a factor of 2 , leading to a $1 \%$ variation only of the total cross section.

In the vibronic network, the maximum number of 16 vibrational levels is necessary. If one reduces this number, for computation simplicity, to only 11 levels, the total cross 
section does not appreciably diminish $\left(41.4 \AA^{2}\right.$ instead of $42.3 \AA^{2}$ ) but the bump of the experimental curve is not reproduced.

The internal reflexion which was first described in the BFG model, corresponds to exit channels on the ionic curves which are energetically forbidden and lead to several round trips as in complex formation. The behavior obtained in this work is similar to the results of Barker (10). Instead of considering an equal mixture of the orbitals of the $\mathrm{Na}$ atom, the cross sections have been calculated for the three entrance channels $\mathrm{Nal} 3 \mathrm{p}_{x}, 3 \mathrm{p}_{y}$, $\left.3 p_{z}\right)$. The total cross-sections at relative energy of $.15 \mathrm{eV}$ are respectively $41.15,43.1$ and $43.8 \AA^{2}$. The energy transfer differential cross section depends strongly upon the prepared state.

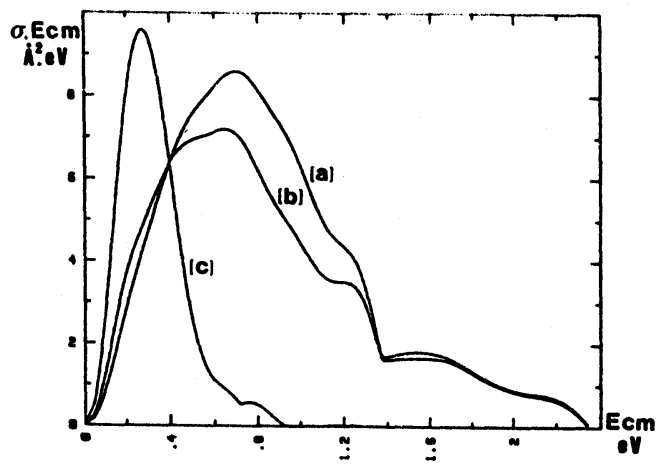

Piqure 5: Differential cross section for the $\mathrm{Na} 3 p_{x}(a), \quad 3 p_{z}(b)$ and $3 p_{y}(c)$ quenching by $0_{2}$.

II. INTERMULTIPLET MIXING AND REACTION.

The cross sections for collisionnal transfers from a given excited state of the 
sodium atom towards the other excited levels, as well as the reaction cross section of these excited states with oxygen molecules have been calculated with the same preceding molecular parameters. For the reacting $4 \mathrm{D}$ state, it varies from $5 \AA^{2}$ at. 1 ev to $250 \AA^{2}$ for .8 eV in good agreement with the experimental observations. The $4 \mathrm{~S}$ state provides a cross section in between 2 and $20 \AA^{2}$ in the same energy range. The main problem of this multiple crossing model is that the $5 \mathrm{~S}$ and the 4D levels have reactive cross sections with the same order of magnitude, in contradiction with experiment. It is possible that for excited states, the exchanged electron behaves as if it were quasi-free. The selection rules for reaction become then those of creation of the $\mathrm{O}_{2}^{-}$, the shape resonance is due to $1=2$ ( $d$ wave) corresponding to a $D$ state in the atomic symmetry group.

The multiple crossing model has been used with success by Gislason for the reaction of ground state alkali atoms with iodine. It is shown here that this model is able to predict the correct total cross section and reproduces the energy differential cross section for the quenching of a first resonant state by oxygen molecules. The intermultiplet quenching cross sections can be also predicted in the case of a large number of excited states involving more than hundred ionic and covalent potential energy curves without any approximation, but for rather highly excited states, this model must be used with caution when the symmetry of the reactants is taken into account. 


\section{REFERENCES}

1

E. A. GISLASON,

J. G. SACHS

J. Chem.

Phys. $\underline{62} 2678(1975)$

2 J.LOS, A.N. KLEYN in Alkali Halide

Vapors Ed.: P. DAVIDOVITS, D. L. MC. FADDEN Academic Press (1979)

3 R. BERSOHN Ed. : P. DAVIDOVITS, D. L. MC. FADDEN Academic Press (1979)

4 I. V. HERTEL, H. HOFMANN, R.A. ROST Chem. Phys. 47163 (1977)

5 J.A. SILVER, N. C. BLAIS, G. H. KWEI J. Chem. Phys. 713412 (1979)

6 Y.T. LEE, J.M. MESTDAGH, to be published

7 E. BAUER, E. R. FISCHER, F. R. GILMORE, J. Chem. Phys. $\underline{51} 4173$ (1969)

8 U. C. KLOMP, M. R. SPALBURG, J. LOS Chem. Phys. 8333 (1984)

9 R. GRICE, D. R. HERSCHBACH Mol. Phys. $\underline{27} 159(1974)$.

10 J. R. BARKER Chem. Phys. 18175 (1976). 